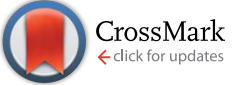

Cite this: RSC Adv., 2015, 5, 67284

\title{
Method to analyse energy and intensity dependent photo-curing of acrylic esters in bulk $\dagger$
}

\begin{abstract}
M. Schmitt
The analysis of the photo-induced polymerization of bulk materials is a very important aspect of scientific and industrial research and applications. Thereby, the interactions between the initiator, the monomer and the light source have to be considered, which have been hardly/not investigated. For example, novel UVlight sources based on LED technology are in the process of development. Hence, the need for such kinetic analyses increases to investigate the multiple energy and intensity dependent influencing factors, like the quantum yield of the primary radical formation and the reactivity's towards double bonds or inhibitors. Within this contribution a dose and wavelength dependent procedure is firstly described in detail, which allows the analysis of the kinetics of the photo-curing and the solidification of multifunctional liquid acrylic esters without a solvent. The photo-polymerizations induced by three different model resins, containing well-known commercial initiators, are monitored by an in situ Raman UV-vis system and analysed in detail. This novel procedure is based on an absolute and spectral calibrated fibre optical receiver, which is part of the Raman UV-vis setup. The multiple influences of the absorbed photons on e.g. the kinetic length of the chain reaction are presented and/or demonstrated. For example, higher yields can be achieved by less energetic and less intense irradiation.
\end{abstract}

Received 15th June 2015

Accepted 21st July 2015

DOI: $10.1039 / \mathrm{c} 5 \mathrm{ra11427f}$

www.rsc.org/advances

analyses of the triple state of the radical reactivity towards

\section{Introduction}

Free radical polymerization is a very familiar method leading to polymers. So, multiple investigations to analyse the reaction kinetics in solution can be found in the literature. ${ }^{\mathbf{1 - 1 0}}$ But the photo-polymerization is an efficient method for the fast production of cross-linked polymers from liquid resins/ monomers without solvents. ${ }^{\mathbf{1 1}, \mathbf{1 2}}$ Solvent-free (= bulk) photopolymerisation is used for the construction of materials and coatings. Our research focuses on the photo-polymerisation of multifunctional monomers, for example as used for printing applications. ${ }^{13-17}$

Nowadays, "printing" is not only a coating procedure; it ranges from coating text or images by UV-printing ${ }^{\mathbf{1 8}}$ (off-set, flexo) or ink-jet printing to construction e.g. by photo-lithography ${ }^{19}$ or $3 \mathrm{D}$ printing. ${ }^{20}$ This topic, including the multiple applications and advantages, was recently reviewed with the focus on LED technology. ${ }^{21}$ Characterization of the kinetics of photo-induced polymerization in bulk is also, from the scientific aspects, an interesting and most important topic., ${ }^{6,13,15,22-29}$ Some aspects that have to be analysed are the identification of the energy/intensity relations resulting in an effective radical formation. This includes the quantum yield determination and

Saarland University, Campus B 2 2, 66123 Saarbruecken, Germany. E-mail: mic. schmitt@mx.uni-saarland.de

$\dagger$ Electronic supplementary information (ESI) available: Additional equations and visualizations of all measurements. See DOI: 10.1039/c5ra11427f acrylate double bonds or inhibition compounds. The presented way to perform energy and intensity variations of the light source may help to analyse these and further effects. Considering applications such as 3D printing or UV-printing the properties of known/used initiators are far from perfect. Multiple problems in aspects of health and/or of efficiency exist. $^{30-37}$

In the past, one process constant within the interplay between initiators, monomer and light source was the light source. For example, for printing applications like off-set or flexo printing a mercury mid-pressure lamp was the only applicable light source (power in the range of $200 \mathrm{~W} \mathrm{~cm}^{-1}$, high intensity in UV-range). Such complex systems have not been or have hardly been investigated because analysing the kinetics of polymerization or solidification without a solvent is a challenging problem, especially if practical orientations are proposed. Nowadays and in the future the light source is, or will be, an additional adjustable parameter.

LED technology can be considered as being well-established in the visible range. ${ }^{21}$ In the UV-range LED's are still in the process of development. Due to the multiple advantages of LED $^{21}$ technology, curing units basing on LEDs are purchasable even today, for example, a high power LED up to $7 \mathrm{~W} \mathrm{~cm}^{-1}$ at $365 \mathrm{~nm} \pm 6 \mathrm{~nm} .{ }^{38}$ This wavelength is smaller than the onset of absorbance of a number of established initiators and some resins. ${ }^{28}$ At the moment no LED's with comparable intensities exist in the main/strong absorption range of most initiators, 
which is located below $365 \mathrm{~nm}$. Hence, the initiators and the initiator monomer combinations cannot be characterized and new systems cannot be developed but "new photo-initiating systems and strategies"21 have to be developed. This lack can be removed by the techniques and procedures presented within the following study.

Recently an UV-vis solidification monitoring system ${ }^{\mathbf{1 4}}$ and the combination of Raman and the UV-vis monitoring system were firstly introduced/discovered. ${ }^{16,17}$ The combination of the Raman with the UV-vis system, Scheme 1, leads to the possibility to investigate the efficiency of initiators in detail. For the present study this system is further varied by a spectral and absolute calibrated light source. ${ }^{39}$ For absolute calibration an absolute and spectral calibrated receiver for a large amount of photons is used. The recently developed procedure will also be explained within the manuscript. The combination of a linear variable filter, LVF and a band pass filter leads to the possibility to vary the energy with comparable intensity and to vary the intensity with constant energy by using filter combinations and by frequency variation.

The main objective of the presented study is a detailed analysis of the novel experimental setup/technique, including dose and energy relations, leading to e.g. kinetic lengths of the chain reaction and different efficiencies. The demonstration of the complex but easily implementable procedure takes place using three commercial, available, well-known and often used initiators. The first one, benzophenone, ${ }^{\mathbf{4 0}} \mathrm{BP}$, is an nonfragmenting, Norrish type II initiator, normally used together with a co-initiator. ${ }^{41}$ The second one, 2-hydroxy-2methylpropiophenone, DMHA, is a versatile highly efficient liquid photo-initiator, which is used to initiate the photopolymerization of chemically unsaturated prepolymers. ${ }^{42}$ The third one, 2,4,6-trimethylbenzoyl-diphenylphosphine oxide, TPO-L, is also a highly reactive/efficient liquid photo-initiator. DMHA and TPO-L react via light absorption, critical activation and the cleavage of a fragile carbon bond, Norrish type I initiator. The model resin is a well-defined but multifunctional inhibitor containing a printable acrylic ester monomer mixture, which is applicable e.g. as a lacquer or finish for off-set printing.

To summarize, the contribution contains the development of, and the demonstration for, wavelength and intensity dependent experimental analysis using a Raman UV-vis setup. This includes a detailed analysis of the measured data, which means the Raman, the UV-vis and the dimensions of the cured solid. An important aspect is the identification of the signal curing correlations of the UV-vis system, even in the "influenced" wavelength range. Recently, only the reflection on the fused silica to resin interface was analysed, no. 3 in Scheme 1. Additionally, a cascading effect of a $45^{\circ}$ incident angle of the Raman UV-vis setup is clearly proven for (semi-)transparent resins. Due to the absolute and spectral calibrated light source together with the novel data analysis, the measurement system has the potential to enhance the analysis of photo-induced polymerizations in bulk. This applies not only for the application aspects but also for practical and theoretical studies concerning the kinetics, quantum yields (quantum yield for triplet, quantum yield of radical formation or radical reactivity towards
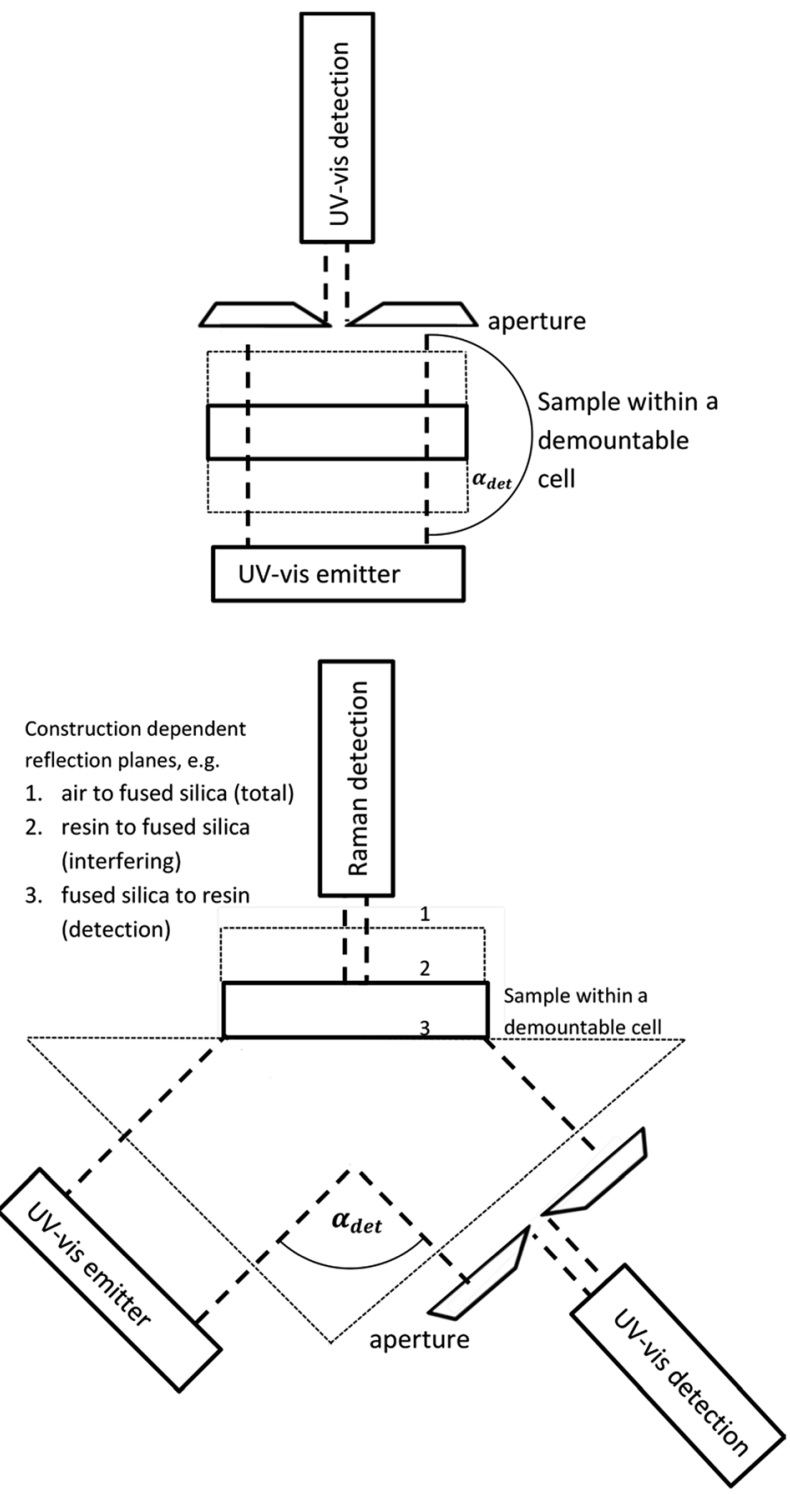

Scheme 1 UV-vis curing transmission (top) and Raman UV-vis geometry (bottom). The fused silica windows and the prism are marked by thin broken lines. If the geometry is alternated (e.g. by using IR transparent material) the FT-Raman system can also be replaced by a FT- or disperse IR spectrometer (compare also to Section 1 within the $\mathrm{ESI} \dagger$ ).

acrylate double bonds or inhibition species) and band gap effects (if using semiconductor based initiators ${ }^{13,16,17}$ ).

\section{Experimental}

\subsection{Materials}

The resin was a well-defined 2 to 3 mixture of trimethylolpropane triacrylate, TMPTA, and bisphenol A diglycidyl ether diacrylate, BAGdA, exactly (1-methylethylidene)bis[4,1-phenyleneoxy(2hydroxy-3,1-propanediyl)]diacrylate received from Rahn AG, Zürich, Switzerland $\left(\langle\rho\rangle=1.148 \mathrm{~g} \mathrm{ml}^{-1}\right)$. The number of double bonds $n_{\mathrm{DB}}$ can be calculated by eqn (1), compare to ESI, $\uparrow$ with known mass $m_{\text {total }}$. 
Table 1 Investigated initiators and amount within the sample

\begin{tabular}{|c|c|c|c|c|c|c|}
\hline Brand name & Name & Formula & $\begin{array}{l}M \\
{\left[\mathrm{~g} \mathrm{~mol}^{-1}\right]}\end{array}$ & $\begin{array}{l}\text { Maximum of } \\
\text { absorbance }^{c}[\mathrm{~nm}]\end{array}$ & $\begin{array}{l}n_{\mathrm{INI}} / n_{\mathrm{C}=\mathrm{C}} \\
{[\mathrm{mol} \%]}\end{array}$ & $\begin{array}{l}\text { Weight content } \\
{[\%]}\end{array}$ \\
\hline $\begin{array}{l}\text { Genocure*} \mathrm{BP}^{a} \\
\text { Irgacure* } \mathrm{BP}^{b}\end{array}$ & Benzophenone & $\mathrm{C}_{13} \mathrm{H}_{10} \mathrm{O}$ & 182.22 & 254 & 1.05 & 1.25 \\
\hline 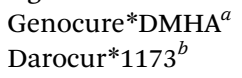 & $\begin{array}{l}\text { 2-Hydroxy-2- } \\
\text { methylpropiophenone }\end{array}$ & $\mathrm{C}_{10} \mathrm{H}_{12} \mathrm{O}_{2}$ & 164.20 & $247 / 277$ & 0.95 & 1.02 \\
\hline $\begin{array}{l}\text { Genocure*TPO-L }^{a} \\
\text { Irgacure*TPO-L }^{b}\end{array}$ & $\begin{array}{l}\text { 2,4,6-Trimethylbenzoyl- } \\
\text { diphenylphosphine oxide }\end{array}$ & $\mathrm{C}_{22} \mathrm{H}_{21} \mathrm{O}_{2} \mathrm{P}$ & 348.37 & 380 & 0.89 & 2.01 \\
\hline
\end{tabular}

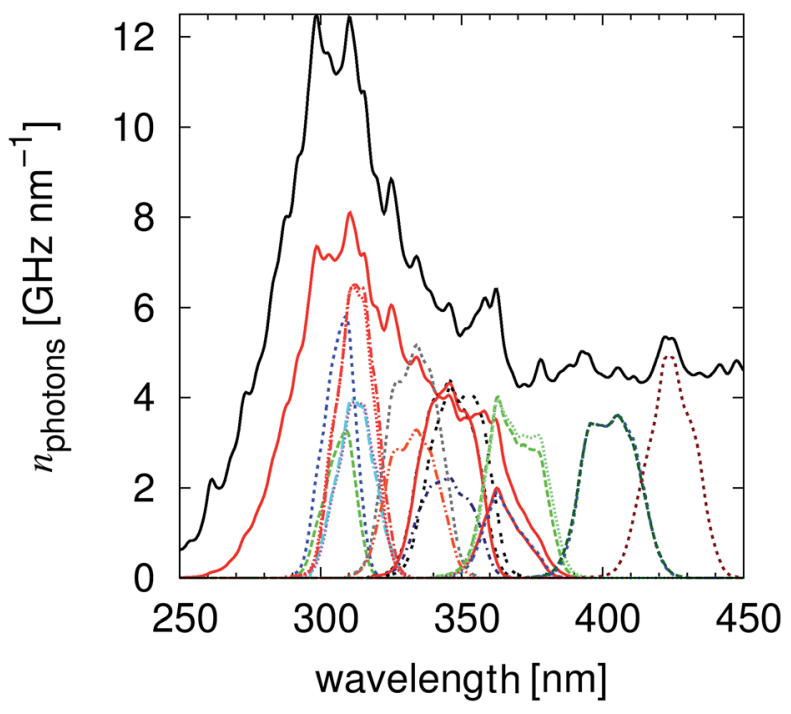

Fig. 1 The calculated irradiated number of photons per $\mathrm{nm}$ for the different filter combinations. The illuminations with the highest impact of the UG11 filter are the measurements performed with the LVF-HL $\approx 370 \mathrm{~nm}$.

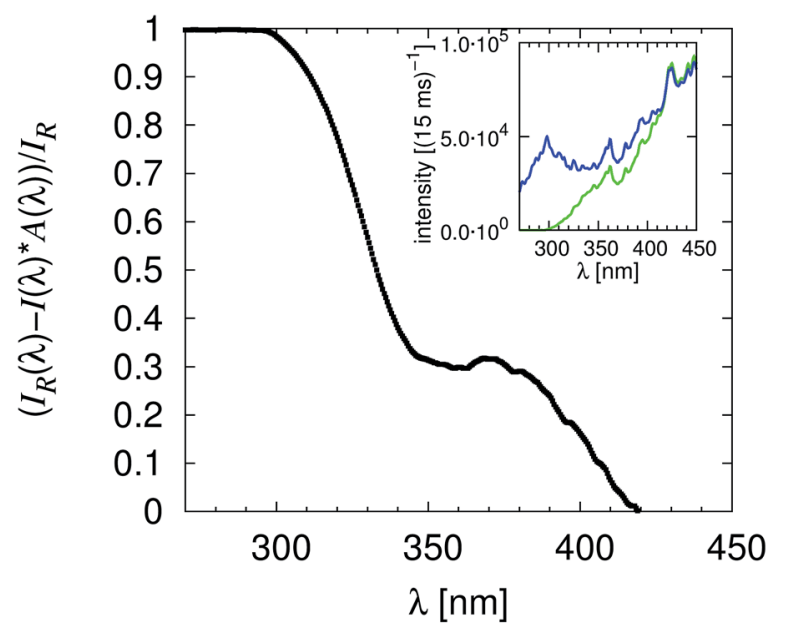

$$
\text { - TPO-L }-I(\lambda) \quad-I_{R}(\lambda)
$$

Fig. 2 Relative absorption of the resin containing 2 wt\% TPO-L $(d=$ $0.05 \mathrm{~mm})$, compare also with Fig. $\mathrm{S} 4 . \dagger$

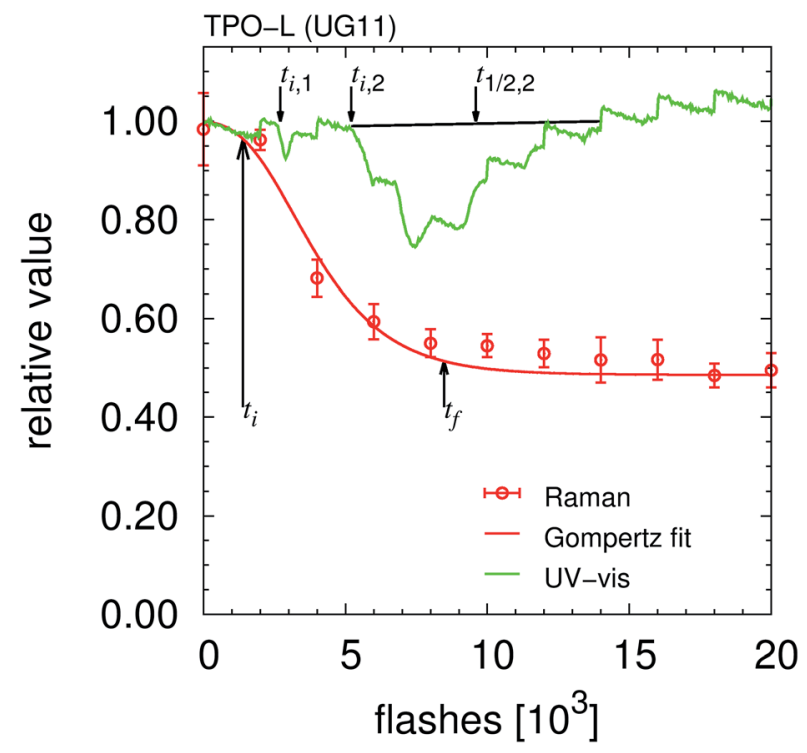

Fig. 3 Visualisation of the relation between the monomer conversion and the detected UV-vis signal variations. All determined "times" are marked within the figure, compare also to Fig. S5. $\dagger$

$n_{\mathrm{DB}}=\frac{N_{\mathrm{DB}}}{N_{\mathrm{A}}}=\left(\frac{3 \times 0.4}{M_{\mathrm{TMPTA}}}+\frac{2 \times 0.6}{M_{\mathrm{BAGdA}}}\right) m_{\mathrm{total}}=\frac{m_{\mathrm{total}}}{153.23} \mathrm{~mol} \mathrm{~g}^{-1}$

The three initiators investigated within this study, Table 1, were also purchased from Rahn AG. The initiators were added/ mixed prior to the first measurement with the monomer.

\subsection{UV-vis and irradiation}

The transmission measurements of the compounds dissolved in isopropyl alcohol, iPA, were performed with a Carry50 Bio, Agilent, Waldbronn, Germany, Fig. S3.† For all other experiments a modular, spectral and absolute calibrated fibre optical system was used. The detection unit was a MultiSpec Desktop/ USB system, tec5 AG, Oberursel, Germany equipped with a MCS FFT-CCD diode array detector, Carl Zeiss, Oberkochen, Germany. The back-thinned FFT-CCD Chip S7031-1006S, 1024 $\times 64$ pixel, was fabricated by Hamamatsu Photonics K.K., Herrsching am Ammersee, Germany. For coupling a 74-UV collimating lens and an optical fibre (core diameter $0.4 \mathrm{~mm}$ ) UV- 
Table 2 Summary of the raw data of all measurements

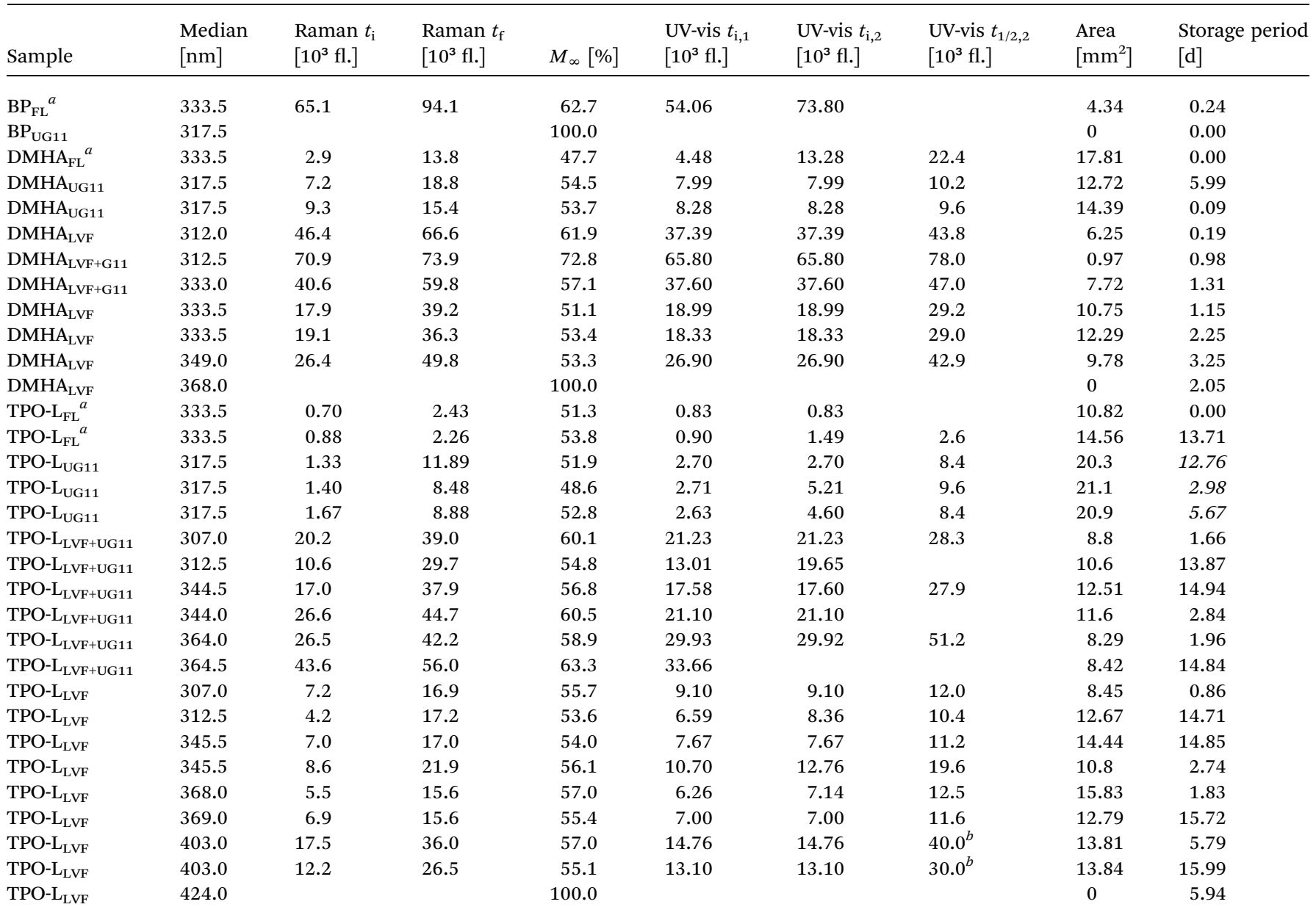

${ }^{a}$ Only wavelengths $<450 \mathrm{~nm}$ are considered for the flash light without a filter (resulting in a median of $333.5 \mathrm{~nm}$ ). The total energy is around $115 \mathrm{~W}$ $\mathrm{m}^{-2}$. The measurement without filter of the TPO-L containing sample was performed with 200 flashes in-between the Raman measurements. ${ }^{b}$ Second signal cannot be identified without any doubt.

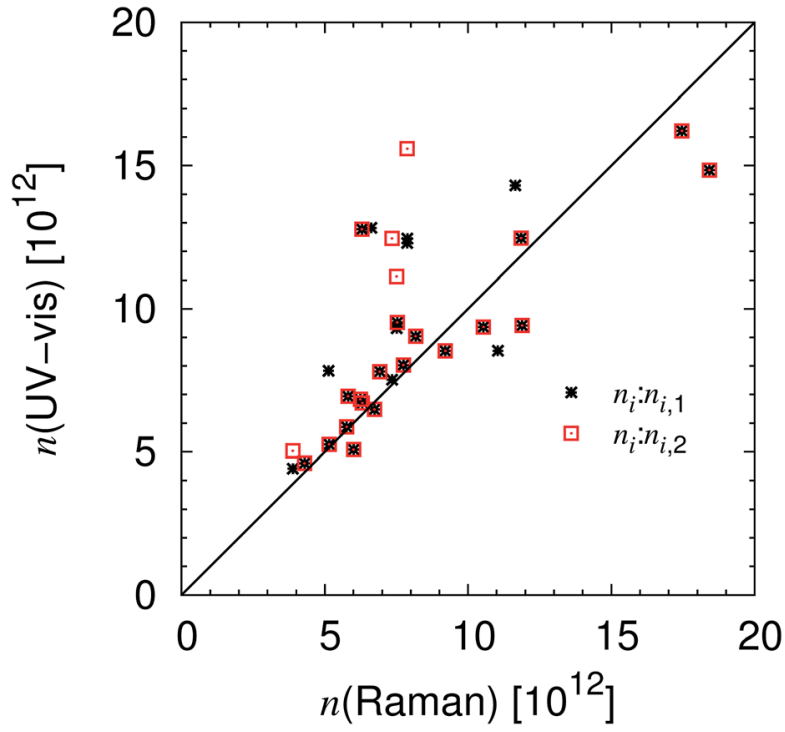

Fig. 4 Initial times for the Raman and the UV-vis setup. The calculated initial times in number of absorbed photons are presented, which are proportional to the number of flashes, compare also to Fig. S6. $\dagger$
VIS XSR solarization-resistant QP400-2-SR-BX from Ocean Optics GmbH, Ostfildern, Germany were used. The fibre optical detector was connected with a PVC-housing containing a 0.15 $\mathrm{mm}$ pinhole or an absorptive neutral density $\mathrm{ND}=3$ (NE30A), Thorlabs Inc., Newton, New Jersey, USA.

This unit can be connected with a Raman UV-vis ${ }^{16}\left(45^{\circ}\right.$ geometry $\left(\alpha_{\text {det }}=90^{\circ}\right)$ ) or a UV-vis sample cell $\left(90^{\circ}\right.$ geometry $\left(\alpha_{\text {det }}=180^{\circ}\right)$, transmission, aligned) for monitoring the photo induced polymerization of bulk acrylic esters, Scheme 1. For illumination, the cells were also connected via optical fibre (core diameter $0.4 \mathrm{~mm}$ ) to a Perkin Elmer optoelectronics xenon flash light (LS-FX) FX 1160 (maximum output 0.5 J per flash, regular $370 \mathrm{~mJ}$ per flash, $\approx 25 \mathrm{~W}$ light output, $8 \mu \mathrm{s}$ per flash, $66.6 \mathrm{~Hz}$ ). The light source is modified in such a way that a linear variable filter and a UG11 filter can be used simultaneously without alternation of the beam path. Both were purchased from Ocean Optics GmbH, Ostfildern, Germany. The linear variable filter, LVF-HL, is an adjustable bandpass filter with the working range of 300 to $750 \mathrm{~nm}$. A specific correction function $A_{\mathrm{c}}(\lambda)$ exists for the detector, 
Table 3 Example of measured and calculated dimensions of/for the solids after curing

\begin{tabular}{|c|c|c|c|c|c|}
\hline & $2 a[\mathrm{~mm}]$ & $2 b[\mathrm{~mm}]$ & $\begin{array}{l}\text { Area measured } \\
{\left[\mathrm{mm}^{2}\right]}\end{array}$ & $\begin{array}{l}\text { Area calculated } \\
{\left[\mathrm{mm}^{2}\right]}\end{array}$ & $\begin{array}{l}\text { Theoretical incident } \\
\text { angle }\left[{ }^{\circ}\right]\end{array}$ \\
\hline TPO-L $307 \mathrm{~nm}$ & 2.65 & 4.02 & 8.45 & 8.37 & 41.2 \\
\hline TPO-L $312 \mathrm{~nm}$ & 2.86 & 5.64 & 12.67 & 12.67 & 30.5 \\
\hline TPO-L (+ UG11) $312 \mathrm{~nm}$ & 2.90 & 4.59 & 10.06 & 10.45 & 39.2 \\
\hline DMHA $312 \mathrm{~nm}$ & 1.97 & 4.06 & 6.25 & 6.28 & 29.0 \\
\hline $\operatorname{Cyan}^{a}$ & 1.82 & 2.63 & 3.75 & 3.76 & 43.8 \\
\hline Magenta $^{a}$ & 1.88 & 2.75 & 4.05 & 4.06 & 43.1 \\
\hline Yellow $^{a}$ & 2.33 & 3.14 & 5.68 & 5.76 & 47.9 \\
\hline Key $^{a}$ & 1.29 & 1.89 & 2.00 & 1.92 & 43.0 \\
\hline
\end{tabular}

${ }^{a}$ These example measurements for non-transparent resins may be found within the ESI, Fig. S7.

$$
E(\lambda)=n(\lambda)_{\text {photon }} \times \frac{h \times c}{\lambda}=n(\lambda)_{\text {counts }} \times A_{\mathrm{c}}(\lambda) \times \frac{h \times c}{\lambda},
$$

which was computed by a sophisticated statistical procedure, ${ }^{39}$ Table S1. $\uparrow$ This function can be used to calculate the average photon number $n(\lambda)_{\text {photon }}$ in $\mathrm{Hz} \mathrm{m}^{-2}$ and the irradiance $E(\lambda)$ in $\mathrm{W} \mathrm{m}^{-2}$ using only the count number $n(\lambda)_{\text {count }}$ per array of the fully illuminated detection area. The function itself is welldefined above $310 \mathrm{~nm}$. Below $310 \mathrm{~nm}$ eqn (3) is used,

Table 4 Integrals of the calculated incident irradiation $n_{\mathrm{ill}}, E_{\mathrm{ill}}$ and relative absorptions of the initiators $n_{|\mathrm{N}|}, E_{|\mathrm{N}|}$ and resin $n_{\mathrm{R}}, E_{\mathrm{R}}$

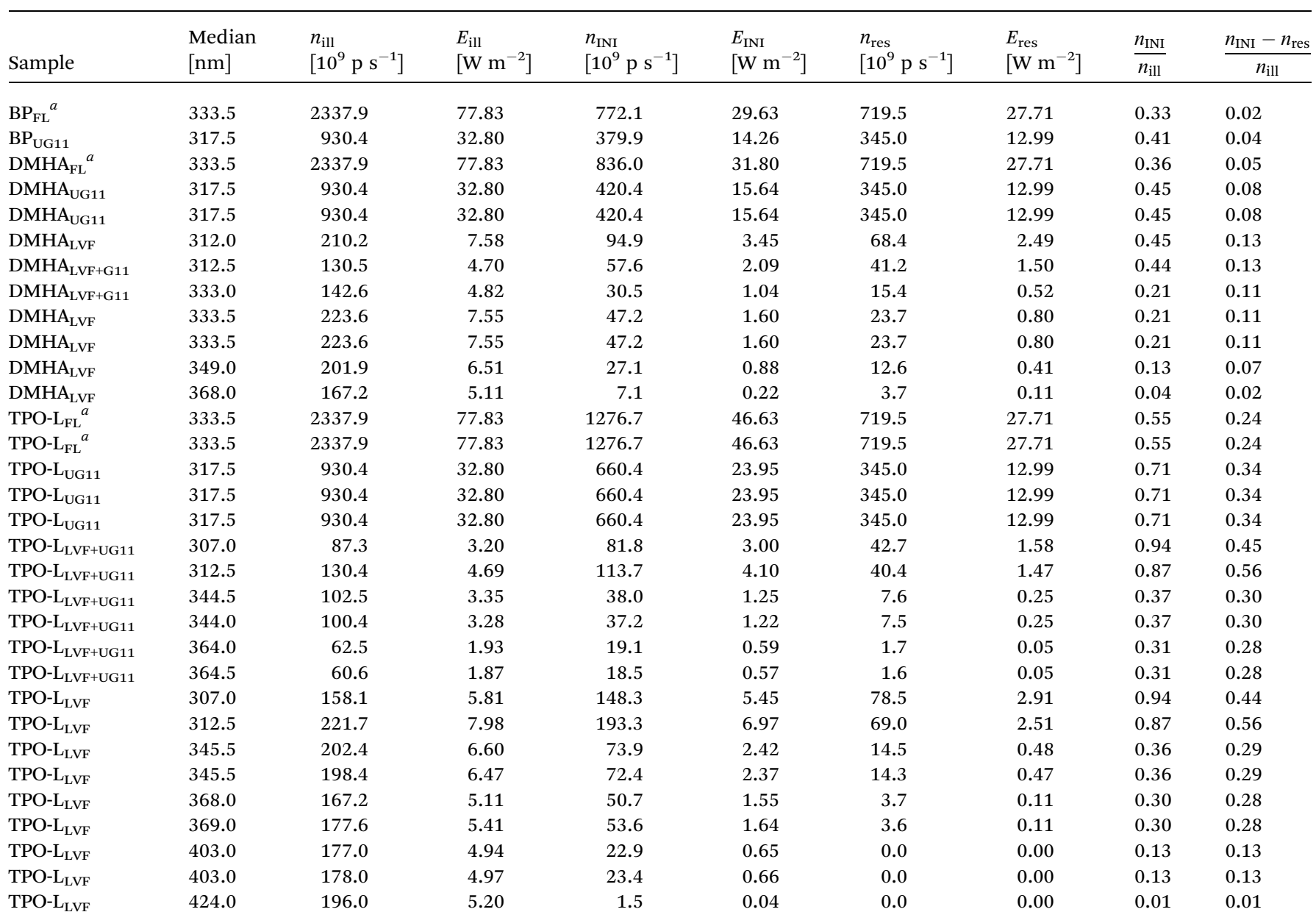

${ }^{a}$ Only wavelengths $<450 \mathrm{~nm}$ are considered for the flash light without filter. The total energy is around $115 \mathrm{~W} \mathrm{~m}^{-2}$. The measurement without filter of the TPO-L containing sample was performed with 200 flashes between the Raman measurements. 


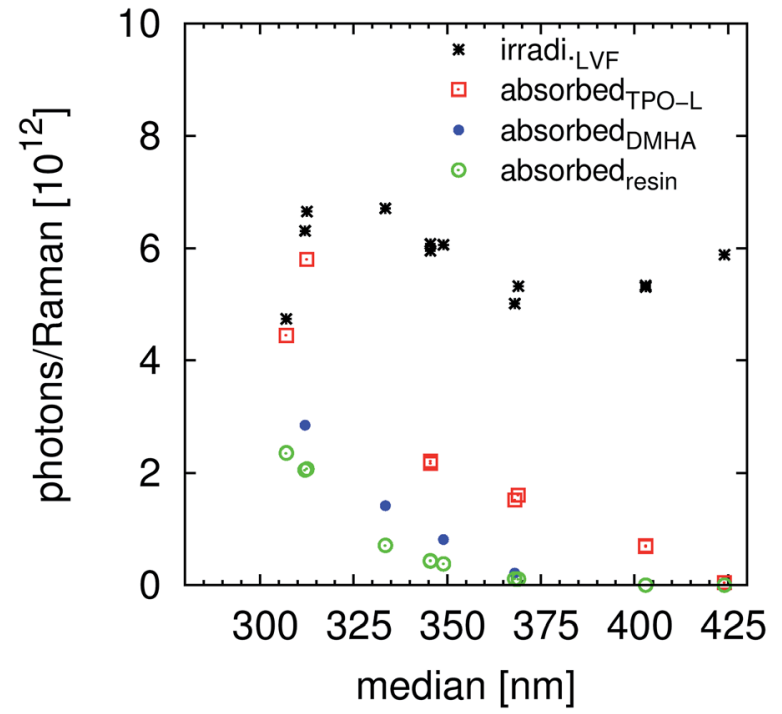

Fig. 5 Overview of the incident irradiation $n_{\text {ill }}$ and the absorbed photons $n$ for the initiators and the resins during one cycle of irradiation between the Raman measurements (LVF-HL). Compare also to the illuminations within Fig. S9.† The number of photons is relative to the calculated illumination area, Table 3.

$$
A_{\mathrm{c}}(\lambda)=A_{\mathrm{c}}(310 \mathrm{~nm})\left(\frac{\lambda-176 \mathrm{~nm}}{310 \mathrm{~nm}-176 \mathrm{~nm}}\right)^{2},
$$

to consider a reduction in the quantum yield of the detection unit. The function $A_{\mathrm{c}}(\lambda)$ is valid if the detection area of the collimation lens $\left(0.41 \mathrm{~mm}^{2}(\approx 75 \%)\right.$ to $\left.1.23 \mathrm{~mm}^{2}(100 \%)\right)$ is sufficiently uniformly illuminated. The xenon flash light is too intensive to be determinable without a filter or pinhole, neither within an aligned ("transition") nor within the Raman UV-vis setup, Scheme 1. The scaling procedure will be described in the subsection "Calculation of the intensity and energy of the irradiated light" (3.6). The light using the ND3 filter or the 0.150 $\mathrm{mm}$ pinhole differs by a factor of $1 / 32$ to $1 / 42$. This value is in the range of the differences in area, see Table 3 of the contribution dealing with the detailed characterization of this fibre optical receiver. ${ }^{39}$ An additional proving of the uniform illumination is performed for an aligned setup and will be part of a further research report.

\subsection{Raman UV-vis cure monitoring}

The sample cell of the Raman UV-vis system consists of PVChousing containing a fused silica isosceles right angle prism and two plugs for the collimator lens or the UV-vis detection; Scheme 1, bottom and Fig. 2 in ref. 16. The plugs are positioned at the centre of the smaller planes to allow illumination and UVvis detection in $\alpha_{\text {det }}=90^{\circ}$ geometry (angle of incidence $=$ angle of detection $=45^{\circ}$ ). The larger (top) plane can be covered with a fused silica window (1 mm), Scheme 1, bottom. At the centre of this plane, in-between the cover and the prism, the liquid samples were placed. The $0.05 \mathrm{~mm}$ thickness was ensured by using a distance holder. Thickness accuracy was controlled, as explained within the discussion in section 3.5 , by quantitative
Raman spectroscopy. All samples were sufficiently transparent to avoid heating due to the absorption of the $1064 \mathrm{~nm}$ laser (1 W) from the Multiram FT-Raman spectrometer (Bruker Optic, Eislingen, Germany). For every vibrational band determination, four Raman spectra $\left(6 \mathrm{~cm}^{-1}\right.$ resolution, average spectrum of 20 measurements, $\approx 1$ min measuring time) of the samples were recorded in back-reflection, Scheme 1, vertically through the cover during light-off phases (in general after every 2.000 flashes). UV-vis data were recorded/averaged during the illumination with $10 \times 4$ flashes. This recently introduced automatic Raman UV-vis system ${ }^{16}$ has the unique ability to monitor both the double bond content (deep curing by Raman) and solidification (area curing by UV-vis). Before the present study, the analysis of the UV-vis data was only performed for wavelength ranges in which the sample contains strong absorbers, leading to non-disturbed reflection at the fused silica to resin interface, no. 3 in Scheme 1. But in this study it will be shown that all wavelength ranges with sufficient illumination intensity can be analysed, see the subsection "Analysis of the UV-vis signal to curing correlation" (3.3). After solidification the still liquid monomer is removed by washing with ethanol and the size of the solid, Fig. S1, $\dagger$ was determined from images of the SM Z800 stereomicroscope coupled with a DS-2Mv CCD camera by the imaging software NIS Elements D, 3.00 from Nikon GmbH, Düsseldorf, Germany.

\subsection{Data processing Raman}

The Raman data were fitted by multiple pseudo-Voigt functions $\operatorname{PV}(\tilde{v})$,

$$
\begin{gathered}
F(\tilde{\nu})=\sum \operatorname{PV}_{\mathrm{i}}(\tilde{\nu}), \\
\operatorname{PV}(\tilde{\nu})=\operatorname{area}(\eta g(\tilde{\nu})+(1-\eta) l(\tilde{\nu})), \\
g(\tilde{\nu})=\frac{4 \sqrt{\ln (2)}}{\omega \sqrt{\pi}} \exp \left(-4 \ln (2)\left(\frac{\tilde{\nu}-v_{\text {peak }}}{\omega}\right)\right), \\
l(\tilde{\nu})=\frac{1}{\pi} \frac{0.5 \omega}{\left(\left(\tilde{\nu}-v_{\text {peak }}\right)^{2}+(0.5 \omega)^{2}\right)^{2}},
\end{gathered}
$$

to obtain the area of each vibrational band, especially the one of the double bonds at around $1635 \mathrm{~cm}^{-1}$. The parameters $\omega, v_{\text {peak }}$, and $0 \leq \eta \leq 1$ correspond to the full width at half maximum, the peak position (in $\mathrm{cm}^{-1}$ ) and the relation between the Gaussian shape, eqn (6), and the Lorentzian shape, eqn (7), which is individually calculated for every band. The specific curve shapes of the relative double bond area $\left(\mathrm{C}=\mathrm{C}_{\text {acrylic }}\right.$ to $\left.\mathrm{C}=\mathrm{C}_{\text {aromatic }}\right)$ as a function of the irradiated light ("time") for the Raman data were analysed by modified Gompertzian functions ( $k$ rate constant, $t_{\mathrm{s}}$ shift time),

$$
\begin{gathered}
f(t)=b-a \exp \left(-\exp \left(-k\left(t-t_{\mathrm{s}}\right)\right)\right), \\
t_{\mathrm{i}}=\frac{k t_{\mathrm{s}}-\ln (1.5+0.5 \sqrt{5})}{k}, \\
t_{\mathrm{f}}=2 \frac{k t_{\mathrm{s}}-\ln (1.5-0.5 \sqrt{5})}{k}-t_{\mathrm{i}} .
\end{gathered}
$$


Table 5 Summary of the calculated numbers of absorbed photons, the Raman $\Delta n$ chain length and the relative absorption efficiency with maximal influence of the monomer absorbance, see also Tables S4 and S5

\begin{tabular}{|c|c|c|c|c|c|c|c|c|c|}
\hline Sample & $\begin{array}{l}\text { Median } \\
{[\mathrm{nm}]}\end{array}$ & $\begin{array}{l}\Delta n \\
\operatorname{Raman}\left[10^{12} \mathrm{p}\right]\end{array}$ & $\begin{array}{l}\Delta n \\
\text { UV-vis }\left[10^{12} \mathrm{p}\right]\end{array}$ & $\begin{array}{l}n_{\mathrm{i}} \text { Raman } \\
{\left[10^{12} \mathrm{p}\right]}\end{array}$ & $\begin{array}{l}n_{\mathrm{i}, 1} \text { UV-vis } \\
{\left[10^{12} \mathrm{p}\right]}\end{array}$ & $\begin{array}{l}n_{\mathrm{i}, 2} \text { UV-vis } \\
{\left[10^{12} \mathrm{p}\right]}\end{array}$ & $\begin{array}{l}\text { Storage period } \\
\text { [d] }\end{array}$ & $\begin{array}{l}\text { Raman } \Delta n \\
\text { chain length }\end{array}$ & $\begin{array}{l}\mathrm{AE}(\min ) \\
{[\%]}\end{array}$ \\
\hline $\mathrm{BP}_{\mathrm{FL}}^{a}$ & 333.5 & 22.6 & & 51.3 & 42.6 & 58.2 & 0.24 & 94 & 2.2 \\
\hline $\mathrm{DMHA}_{\mathrm{FL}}{ }^{a}$ & 333.5 & 21.8 & 36.8 & 5.1 & 7.8 & 23.2 & 0.00 & 117 & 5.0 \\
\hline DMHA $_{\text {UG11 }}$ & 317.5 & 11.3 & 4.3 & 8.2 & 9.0 & 9.0 & 5.99 & 261 & 8.1 \\
\hline DMHA $_{\text {UG11 }}$ & 317.5 & 8.6 & 3.7 & 10.5 & 9.4 & 9.4 & 0.09 & 244 & 8.1 \\
\hline $\mathrm{DMHA}_{\mathrm{LVF}+\mathrm{UG} 11}$ & 333.0 & 4.5 & 4.4 & 9.2 & 8.5 & 8.5 & 1.31 & 526 & 10.6 \\
\hline $\mathrm{DMHA}_{\mathrm{LVF}}$ & 333.5 & 5.7 & 5.5 & 6.3 & 6.7 & 6.7 & 1.15 & 630 & 10.5 \\
\hline $\mathrm{DMHA}_{\mathrm{LVF}}$ & 333.5 & 5.8 & 7.2 & 6.7 & 6.5 & 6.5 & 2.25 & 117 & 10.5 \\
\hline $\mathrm{DMHA}_{\mathrm{LVF}}$ & 349.0 & 5.2 & 7.2 & 5.8 & 5.9 & 5.9 & 3.25 & 261 & 7.2 \\
\hline $\mathrm{DMHA}_{\mathrm{LVF}}$ & 368.0 & & & & & & 2.05 & & 2.1 \\
\hline TPO- $\mathrm{L}_{\mathrm{FL}}{ }^{a}$ & 333.5 & 15.8 & & 5.8 & 6.9 & 6.9 & 0.00 & 159 & 23.8 \\
\hline TPO-L L LF+UG11 & 307.0 & 9.9 & 7.4 & 11.9 & 12.5 & 12.5 & 1.66 & 256 & 44.8 \\
\hline TPO-L $\mathrm{L}_{\mathrm{LVF}+\mathrm{UG} 11}$ & 312.5 & 20.3 & & 11.6 & 14.3 & 21.6 & 13.87 & 131 & 56.2 \\
\hline TPO-L L LVF+UG11 & 344.5 & 10.7 & 10.5 & 7.7 & 8.0 & 8.0 & 14.94 & 204 & 29.7 \\
\hline TPO-L L LVF+UG11 & 344.0 & 8.1 & & 11.9 & 9.4 & 9.4 & 2.84 & 275 & 29.6 \\
\hline TPO-L $\mathrm{L}_{\mathrm{LVF}+\mathrm{UG} 11}$ & 364.0 & 4.0 & 10.8 & 6.9 & 7.8 & 7.8 & 1.96 & 600 & 27.8 \\
\hline TPO-L $\mathrm{L}_{\mathrm{LVF}+\mathrm{UG} 11}$ & 364.5 & 3.5 & & 11.0 & 8.5 & & 14.84 & 521 & 27.9 \\
\hline TPO-L $\mathrm{L}_{\mathrm{LVF}}$ & 307.0 & 11.3 & 6.6 & 7.5 & 9.5 & 9.5 & 0.86 & 200 & 44.1 \\
\hline TPO-L $\mathrm{L}_{\mathrm{LVF}}$ & 312.5 & 24.4 & 7.6 & 7.9 & 12.3 & 15.6 & 14.71 & 107 & 56.1 \\
\hline TPO-L $\mathrm{LVFF}_{\mathrm{L}}$ & 345.5 & 8.9 & 6.3 & 6.2 & 6.8 & 6.8 & 14.85 & 293 & 29.3 \\
\hline TPO-L $\mathrm{L}_{\mathrm{LVF}}$ & 345.5 & 10.2 & 10.6 & 7.5 & 9.3 & 11.1 & 2.74 & 274 & 29.3 \\
\hline TPO-L ${ }_{L V F}$ & 368.0 & 6.7 & 7.0 & 3.9 & 4.4 & 5.0 & 1.83 & 389 & 28.1 \\
\hline TPO-L $\mathrm{L}_{\mathrm{LVF}}$ & 369.0 & 6.8 & 7.1 & 5.1 & 5.3 & 5.3 & 15.72 & 358 & 28.2 \\
\hline
\end{tabular}

${ }^{a}$ Only wavelengths $<450 \mathrm{~nm}$ are considered for the flash light without filter. The total energy is around $115 \mathrm{~W} \mathrm{~m}^{-2}$. The measurement without filter of the TPO-L containing sample was performed with 200 flashes between the Raman measurements. ${ }^{b}$ Only partially cured, compare to ESI.

This allows for the automatic computation of the time when the reaction starts, $t_{\mathrm{i}}$, (affected by inhibition and oxygen) and the time when the reaction is essentially completed, $t_{\mathrm{f}}$ (the system is solidified). The term $1-a / b$ (from limit value consideration) denotes the relative final monomer content $M_{\infty}$ for Raman measurements. Details concerning the Raman analysis like aspects of noise reduction may be found in the literature..$^{13,15-17,28}$ Due to the used filters the UV-vis data cannot be analytically fitted, ${ }^{16}$ see the subsection "Analysis of the UV-vis signal to curing correlation" (3.3).

\section{Results and discussions}

This "Results and discussions" section can be divided in three main parts. The analysis of the sample and the "light sources" (3.1), together with the experimental curing results (3.2), are the requirements for the analysis of the novel experimental and analytical technique (3.3-3.6). The utilization of a FT-Raman unit is not been mandatory. The vibrational band analysis was performed to verify the UV-vis signal curing correlations (3.3). A second focus is on the calculation of the absolute number of absorbed photons (3.6) using the computed properties of the samples and the "light sources". The detailed analysis is only possible if geometric and experimental influencing factors are considered (3.4 and 3.5). Resin-initiator-light source relations for these model samples were computed and summarized within the last part (3.7 and 3.8). These sections also contain different possibilities to visualize/analyse the results. All experimental details concerning the reactivity of the initiators may be found within Tables S2, S4 and S5. $\dagger$

\subsection{Analysis of the samples and the "light sources"}

UV-vis emission. The intensities passing the linear variable filter $(\approx 100$ spectra), the UG11, the linear variable plus UG11 filter $(\approx 100$ spectra $)$ and without a filter are collected in $180^{\circ}$ geometry (aligned, Scheme 1, top), Fig. S2, $\dagger$ to allow the calculation of the irradiation to be possible. Before applying the sample onto the Raman UV-vis prism, Scheme 1, bottom, the total reflected light $I_{\operatorname{tr}}(\lambda)$ was measured. Linear scaling $(C)$ to match with the aligned spectra and comparing with the xenon 

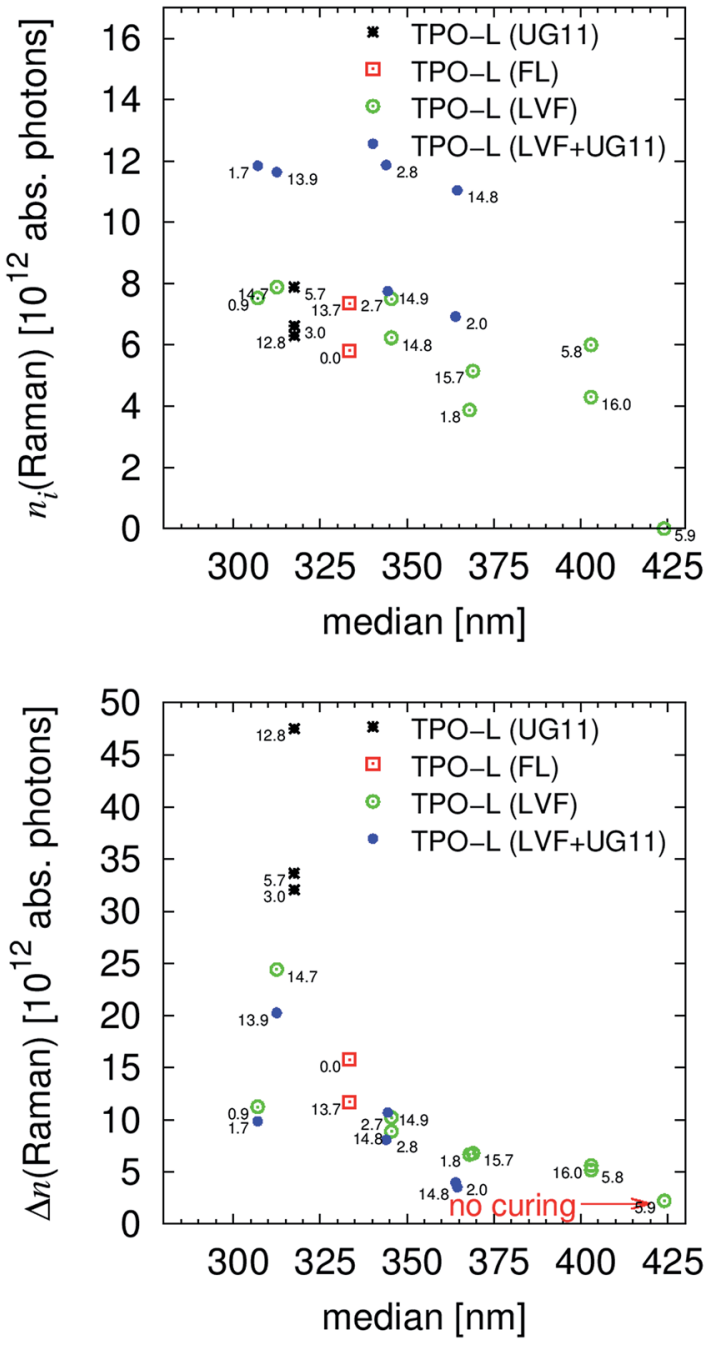

Fig. 6 Visualization of the relations between the illumination wavelength and the absorbed photon number to compensate the inhibition and to cure the resin (efficiency, eqn (15)), compare also to Table 5 and Fig. S10 and S11. $\dagger$ The storage period is labelled within the figure. The storage times have no simple correlation with the curing results, compare to the LVF + UG11 measurements.

light spectrum without a filter, $I_{\mathrm{Xe}, \mathrm{r}}(\lambda)$, allows the calculation of the absolute transmission $T_{\text {ill }}(\lambda)$,

$$
T_{\mathrm{ill}}(\lambda)=\frac{I_{\mathrm{tr}}(\lambda) C}{I_{\mathrm{Xe}, \mathrm{r}}(\lambda)},
$$

which will be used to compute the absolute irradiation $I_{\mathrm{abs}}(\lambda)$, see subsection "Calculation of the intensity and energy of the irradiated light” (3.6), Fig. 1.

The width (upper quartile minus lower quartile) of the irradiation light passing the linear variable filter is, with $11 \mathrm{~nm} \pm 2$ $\mathrm{nm}$, so small that the refractive index influences (Sellmeierian equation) $)^{43}$ can be neglected. In this way, multiple "light sources" with similar and known intensity and energy were realized, Fig. 1.

UV-vis transmission and curing transmission. Determination of the absorbance in non-absorbing solvents (pure iPA)

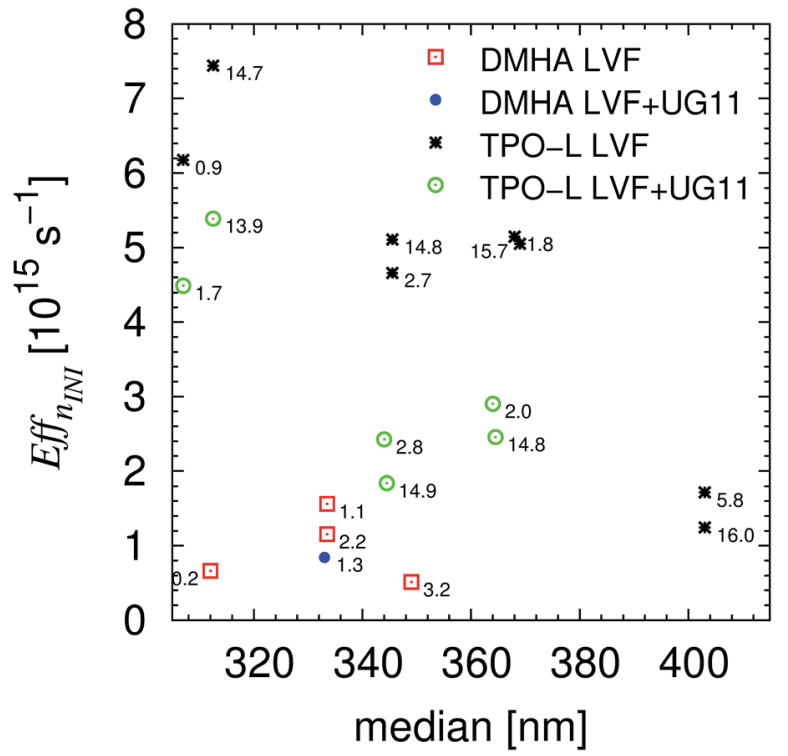

Fig. 7 Relative efficiencies, Eff $n_{\left.n_{|N|}\right|^{\prime}}$ with maximal resins correction of the performed experiments with linear variable filter and linear variable filter plus UG11.

took place to verify the positions given by the distributer, Table 1. For BP and DMHA an additional absorption at $\approx 340 \mathrm{~nm}(A \approx$ $0.8 ; \approx 1.1$ ) was monitored for $1 \mathrm{M}$ solution, Fig. S3. $\dagger$ For these solutions the absorbance is larger than 1 below $300 \mathrm{~nm}$. For $1 \mathrm{M}$ TPO-L solution the absorbance is larger than 1 below $340 \mathrm{~nm}$. For $0.1 \mathrm{M}$ solution a maximum at around $300 \mathrm{~nm}$ was monitored. Transition UV-vis solidification experiments, Scheme 1, top, (thickness $0.05 \mathrm{~mm}$ ) were performed to determine the optical absorption of the initiators and the resin and to compute the absorbed light dose. Only one measurement per sample, resulting in solid polyacrylic esters of around $7.0 \mathrm{~mm}$ diameter, was performed so that no further analysis will be presented. Following these real time solidification measurements, TLO-L, which also has the strongest absorbance within the investigated wavelength range, Fig. $\mathrm{S} 3, \dagger$ results in the fastest photo-polymerisation of the used acrylic ester mixture. The wavelength dependent measurements firstly presented within this study can be used to analyse some of the influencing factors (in addition to absorbance) like the quantum yield for triplet, quantum yield of radical formation or radical reactivity towards acrylate double bonds. The spectral correction procedure firstly introduced in 2011 (ref. 14) to remove the effects of the differences in reflection and refractive index with and without sample, when measuring with the fibre optical system, was performed to determine the relative absorption of the initiators and of the resin, Fig. 2 and S4. $\dagger$ Thereby the relation between the transmitted intensity with, $I(\lambda)$, and without, $I_{\mathrm{r}}(\lambda)$, sample was analytically analysed by eqn (12),

$$
\frac{I(\lambda)}{I_{\mathrm{r}}(\lambda)}=A(\lambda)=a+b \times \lambda+c \times \lambda^{2},
$$

in the range in which no absorbance takes place $(>400 / 450 \mathrm{~nm})$. This procedure leads to the relative absorption $A_{\mathrm{b}}(\lambda)$, 


$$
A_{\mathrm{b}}(\lambda)=\frac{I_{\mathrm{r}}(\lambda)-I(\lambda) A(\lambda)}{I_{\mathrm{r}}(\lambda)} .
$$

Dependencies from a number of not exactly controlled experimental parameters are likely, which can be reduced/ crosschecked by combining these results with the ones of the transmission experiments, Fig. S3.†

\subsection{Experimental results of the Raman UV-vis curing experiments}

Wavelength dependent curing experiments were performed with irradiations passing the UG11 filter, the linear variable filter (different positions), the linear variable filter and the UG11 filter and with no filter at all, Fig. 1. Every Raman spectrum was analysed by the procedure described in subsection (2.4). The area's, eqn (4) to (7), from the Raman spectra, which were collected for every step in irradiation, were averaged. These time/flash dependent area's were analysed by eqn (8) resulting in the final $t_{\mathrm{f}}$, initial time $t_{\mathrm{i}}$ and the final monomer content $M_{\infty}$, Fig. 3. The UV-vis data were analysed as explained within the next subsection (3.3). The median of the illumination light is used for sample nomenclature of the data e.g. within Table 2 . The procedure to calculate the median is explained in the subsection "Calculation of the intensity and energy of the irradiated light" (3.6). The raw data presented in Table 2 clearly demonstrate that the TPO-L as a cleavable photo-initiator is the most reactive initiator. The initiator benzophenone, which works through electron transfer, triple state quenching, ${ }^{44}$ results in a low yield and is the slowest initiator by far.

\subsection{Analysis of the UV-vis signal to curing correlation}

Recently it was demonstrated that the light within strong absorbing ranges of a sample that is reflected at the prism to resin interface can be automatically analysed, resulting in the area solidification analysis of the system, Scheme $1 .{ }^{16}$ Due to the linear variable filter and the tested initiators such unaffected ranges do not exist in this study. Nevertheless, the CCD detector monitored up to two irregular deviations for reflected light, Fig. 3. The number of measurements was too small, so all of the presented hypotheses within this subsection have to be verified by future experimental and theoretical studies. Only a manual determination of two initial times $t_{\mathrm{i}, 1}, t_{\mathrm{i}, 2}$ and the "half effect" time $t_{1 / 2,2}$ was possible. The last one is used to calculate the final time respective to the final absorbed photon number $n_{\mathrm{f}, 2}$,

$$
n_{\mathrm{f}, 2}=n_{\mathrm{i}, 2}+2\left(n_{1 / 2,2}-n_{\mathrm{i}, 2}\right) .
$$

In general, the first irregular deviation $t_{\mathrm{i}, 1} / n_{\mathrm{i}, 1}$ is measured at the start of or before the Raman observation. This means it can be correlated well with the initial time for the Raman measurements, Fig. 3, S5 $\dagger$ and 4. Both UV-vis signals were clearly recognisable for measurements with a high number of absorbed photons (TPO-L UG11, DMHA FL) and in the cases of strong resin absorbance (TPO-L $<312.5 \mathrm{~nm}$ ), Table 2. Then the first effect is less wide and intense than the second UV-vis signal, Fig. 3 and S5. $\dagger$ But at the moment no one-to-one correlation between the initial times of the UV-vis $t_{\mathrm{i}, 1}$ and $t_{\mathrm{i}, 2}$ and the Raman observations $t_{\mathrm{i}}$ can be proven beyond doubt. The same is valid for photo-polymerisation efficiency $\Delta n$,

$$
\Delta n=n_{\mathrm{f}}-n_{\mathrm{i}},
$$

Fig. S5 $\dagger$ and 3. For wavelength ranges smaller than $350 \mathrm{~nm}$ the efficiency $\Delta n$ of the UV-vis data is smaller than the one of the Raman data and for ranges larger than $350 \mathrm{~nm}$ vice versa. This implies that the penetration depth, which significantly influences the Raman measurement (deep curing), has a smaller impact on the UV-vis setup. Hence the UV-vis signal variation is, even for these (partially) transparent wavelength ranges, a suitable probe to monitor the curing of a thin layer. Two aspects that may influence the comparability of the data are the different local positions, see subsection (3.4), and the different resolutions of the equipment (resolution Raman $=2000$ flashes $\gg 40$ flashes $=$ resolution UV-vis). But the present study confirms without any doubt that the variation in the UV-vis signal is correlated with the photo-induced polymerization of the resin. This is the most important point of this subsection.

To emphasize, the $45^{\circ}\left(\alpha_{\mathrm{det}}=90^{\circ}\right)$ reflection UV-vis setup, Scheme 1, is even applicable for transparent systems but its main advantage is the fact that non-transparent systems, like highly pigmented systems, can be analysed.

Additionally, the change in refractive index, which causes the signal variation, is correlated with the solidification of the system and the UV-vis equipment has a better resolution with 40 flashes (possible down to 1 flash) than the Raman equipment (2000 flashes). Additionally, the UV-vis system allows the performance of real-time (this detector down to $3 \mathrm{~ms}$ ) of multiple data points (every $0.5 \mathrm{~nm}$ ). All of these multiple data points specifically vary during the measurement whereas specific vibrational bands measured e.g. by disperse infrared $\left(810 \mathrm{~cm}^{-1}\right)^{23,24}$ or Raman spectroscopy $\left(1635 \mathrm{~cm}^{-1}\right)$ consist only of a few evaluable data points.\$ Further, the UV-vis setup, Scheme 1, is not restricted to transmission experiments. Both $45^{\circ}\left(\alpha_{\mathrm{det}}=90^{\circ}\right)$ and $90^{\circ}\left(\alpha_{\mathrm{det}}=180^{\circ}\right)$ were measured and analysed. The "always" present light source can be used for curing and monitoring. Last but not least the fused silica is much easier to handle/clean than materials that are transparent for IR-radiation. By varying the construction a similar UV-vis receiver can also be connected with any real-time IR spectrometry, see also the ESI. $\dagger$

\subsection{Analysis geometry effects of the setup}

Geometry effects: position. Analysis of the data, Table 2, confirms that the Raman and UV-vis positions are within the area of maximal intensity. The measurement of the DMHA containing system with an irradiation median of $312.5 \mathrm{~nm}$ results in an area of $0.97 \mathrm{~mm}^{2}$. The measurable signal changes both for the UV-vis and the Raman setup. This fact clearly underlines the good match between the UV-vis and the Raman measuring position. This is also the position of maximal

$\ddagger$ Details concerning the UV-vis, Raman and IR may be found within the ESI $\dagger$ and within literature, Fig. 4 in ref. 16. 
intensity, which will be less influenced by reflection effects, compared with the influence of the incidence angle, see below.

Geometry effects: volume. For quantum yield calculation the number of double bonds have to be known. Hence the detection volume has to be specified. Due to the illumination (Scheme 1, bottom) the volume is considered as a $45^{\circ}$ prism (height 0.05 $\mathrm{mm}$ ) whereby the diameter of the base area ranges from $\approx 0.15$ $\mathrm{mm}$ (laser) to $0.20 \mathrm{~mm}$ (collected by the pinhole). For the calculations, Fig. 4, the minimal diameter is chosen. Then the amount of sample/double bonds within the curing volume can easily be calculated from the data by eqn (1). Note that for quantum yield calculation the uncertainty in the area is without any impact (photon number is in $\mathrm{Hz} \mathrm{m}^{-2}$ ). The consumed double bonds are calculated by multiplying with the conversion $\left(1-M_{\infty}\right)$, Table 2 . Note that also the double bond content for every position in time can be determined by eqn (8).

Geometry effects: incidence angle. In the ideal case the incidence angle of the irradiation with $45^{\circ}$ leads to an elliptical cured solid with the well-defined aspect ratio of $2^{-0.5}$. Within the first presentation of the Raman UV-vis system ${ }^{\mathbf{1 6}}$ a second curing area was described, which was monitored for high-yield initiators (or a huge number of flashes) by testing sufficient transparent resins. This second curing area is affected by the light total-reflected at the cover window air interface, no. 1 in Scheme 1 . The detailed analysis of the areas within this study leads to the fact that a cascading effect of the irradiated light within semi-transparent systems influences the dimension of the cured solid significantly even if no second curing area exists. For visualization a theoretical incident angle $\theta_{\mathrm{i}}$ is calculated by eqn (16),

$$
\sin \left(\theta_{\mathrm{i}}\right)=\frac{2 a}{2 b}
$$

The area $A_{\text {ell }}$ can also be calculated with the determined axis $a$ and $b$,

$$
A_{\mathrm{ell}}=\pi a b
$$

to prove the geometric form of the cured solid, Table 3. The results confirm that the first curing area is elliptical. If the system is sufficiently transparent a cascading effect, such as the light (multi-)reflected at the resin to fused silica interfaces, no. 2 in Scheme 1, is clearly recognizable by the theoretical incident angle $\ll 45^{\circ}$. For non-transparent systems (CMYB; highly filled resins), respective for wavelengths within the optical absorption of the monomer/polymer, for example experiment TPO-L (+ UG11) $307 \mathrm{~nm}$, the effect does not exist. By analysing the data, even a distinction between the absorption effectivity of the DMHA and the TPO-L relative to the one of the resin is possible. Hence the measured areas are strongly influenced by the reflections on the resin to fused silica interface, no. 2 in Scheme 1. The geometry of the solid is most likely also influenced by the width of the beam relative to the sections of the linear variable filter. Concerning the area analysis, also the effects of the variance in layer thickness cannot be separated, see the section "Fine correction of the layer thickness and resin aging" (3.5).
Nevertheless the area of the cured solid, Fig. S8 $\dagger$ and Table 2, can be used to predict the effectivity of the initiator for lowintensity illumination in combination with the cascading effect, if the total number of flashes for the experiment is similar, Table S2. $\dagger$ From practical aspects this lower intensity in combination with the effects of the (semi-) transparency leads to the possibility to identify highly reactive systems. These systems lead to an effective reaction with low photon flux (power). Hence the overall curing process of industrial applications may be optimised by the variation of the exposure time.

\subsection{Fine correction of the layer thickness and resin aging}

The further analysis of the data without correction led to a conflicting result that the curing of the DMHA containing resin (using UG11) started earlier but was finished later, 6 days after preparation. Hence, in this case, the layer thickness has to be considered. Fortunately, the used Raman setup allows control of the accuracy of the layer thickness by comparing the computed intensities of the maximum of the double bond vibrational band $I$ at $1635 \mathrm{~cm}^{-1}$ before the first irradiation. These intensities are more or less randomly distributed in time whereby for the resins containing TPO-L the average value is $\langle I\rangle=0.038 \pm 0.004$ for the first measurement cycle $(t<6 \mathrm{~d})$ and for the second measurement cycle $\langle I\rangle=0.036 \pm 0.002(t>12.5$ d). Considering the results of the curing experiment using the UG11 filter it can be assumed that the resin is slightly aged. For the two measurements of the DMHA contacting resin with the UG11 filter the difference in Raman intensity is $\Delta I=0.012$, which corresponds to a significant thickness variation. For moderate/sufficient absorbed photons the correction of the final amount $n_{\mathrm{f}}$ and the initial amount $n_{\mathrm{i}}$, by eqn (18)

$$
n_{\mathrm{f}}=\frac{\langle I\rangle}{I}\left(n_{\mathrm{f}, 0}-n_{\mathrm{i}}\right)+n_{\mathrm{i}}
$$

leads to a higher accuracy of the measured curing behaviour, see the section "Intensity and energy relations of the initiators" (3.7).

\subsection{Calculation of the intensity and energy of the irradiated light}

The absolute and spectral calibration of the fibre optical detector led to a correction function $A_{\mathrm{c}}(\lambda)$, eqn (2), which is valid if the whole detection area is illuminated by a uniformly distributed light source ${ }^{39}$ and correlates the count number of the detection unit with the photon number per array. Within the cited publication the specific energy is calculated by trapeze formula,

$$
\text { Value }=0.5 \sum\left(\operatorname{value}\left(\lambda_{i}\right)+\operatorname{value}\left(\lambda_{i-1}\right)\right) \times\left(\lambda_{i}-\lambda_{i-1}\right)
$$

Here the number of photons is calculated by trapeze formula. The general technique to transfer/calculate necessary spectra was described within the cited publication in detail. ${ }^{39}$ First of all the spectra of the illumination xenon light source passing a ND3-filter (aligned geometry) and a pinhole $(0.15 \mathrm{~mm})$ are recorded. Note that the effective beam width (diameter of 
the cured solids) for the aligned geometry is $0.3 \mathrm{~mm}$ broader due to the difference in the construction/distances, Scheme 1. The pinhole spectrum is multiplied by a scalar factor to match with the ND3 spectrum within the transmission range. A detailed study is in preparation that deals with the aligned transition UV-vis setup, Scheme 1, top, and the area distribution of the irradiation light source. First evaluations prove that the intensity of the used xenon flash light is sufficiently uniformly distributed within the receiver detection "area". ${ }^{39}$

Also, no variations in the energy distribution were observable within this "area" (the relative count numbers between different wavelengths do not change). Hence, the absolute irradiation $I_{\text {abs }}(\lambda)$ is allowed to be calculated simply by multiplying with the correction function $A_{\mathrm{c}}(\lambda)$, eqn (2) and Fig. 4,

$$
I_{\mathrm{abs}}(\lambda)=n(\lambda)_{\text {counts }} \times A_{\mathrm{c}}(\lambda) .
$$

The number of irradiated photons effecting the photopolymerization is influenced by the cascading effect $=$ multireflection on the resin to fused silica interfaces (3.4). But it can be assumed that within the centre of the cured solid the effect is relatively small. Hence, absolute photon dependent analysis of the border regions with the $45^{\circ}\left(\alpha_{\mathrm{det}}=90^{\circ}\right)$ setup, Scheme 1 , is not possible. Within the detection area the xenon flash-light source (25 W light output) illuminated the sample with $115 \mathrm{~W} \mathrm{~m}^{-2}$ (176 nm to $\left.990 \mathrm{~nm}\right)$. Due to the energy distribution the value corresponds to $77 \mathrm{~W} \mathrm{~m}^{-2}=1.32 \times 10^{20}$ photons $\mathrm{s}^{-1} \mathrm{~m}^{-2}$ for wavelengths smaller than $450 \mathrm{~nm}$. Following to Fouassier et al., $1993,{ }^{6}$ an $80 \mathrm{~W} \mathrm{~cm}^{-1}$ ( $\mathrm{Hg}$ midpressure lamp) leads to $10^{22}$ photons $\mathrm{s}^{-1} \mathrm{~m}^{-2}$. The presented calculation leads to reasonable results because the used resin, without added initiator, is not curable by the xenon lamp ( $<140000$ flashes) whereas a $0.05 \mathrm{~mm}$ thick layer is solidified after some runs through our $100 \mathrm{~W} \mathrm{~cm}^{-1}(1.5 \mathrm{~kW} \mathrm{Hg}$ midpressure lamp) lab curing unit.

Calculation of the number of absorbed photons. It is well known that, in general, for a transition from the highest occupied molecular orbital to an unoccupied molecular orbital one photon is necessary. Hence it is reasonable to analyse the measurements in terms of the absorbed photons per array $n(\lambda)$,

$$
n(\lambda)=I_{\mathrm{abs}}(\lambda) T_{\mathrm{ill}}(\lambda) A_{\mathrm{b}}(\lambda)=n_{\mathrm{ill}}(\lambda) A_{\mathrm{b}}(\lambda)
$$

where $I_{\mathrm{abs}}(\lambda), A_{\mathrm{b}}(\lambda), T_{\mathrm{ill}}(\lambda)$ and $n_{\mathrm{ill}}(\lambda)$ are the calculated absolute irradiation, the relative absorption, the absolute transmission and the incident irradiation/photon number, respectively, Table 4. By comparing the absolute absorbed number of photons, which is calculated from $n(\lambda)$ by eqn (19), the significance/effect of the number of incident photons is reduced. Hence, the effect of molecular effectiveness can be monitored. Within Fig. 5 the absolute absorbed numbers of photons for the initiators and for the resin are presented.

The absorption efficiency of the monomer/polymer may differ from the one of the initiators but the resin definitively reduces the number of photons absorbable by the initiator, as proven by comparing Table S4 and S5. $\dagger$ Most data discussed within the manuscript are corrected with a maximal influence of the resins, e.g. the relative absorption efficiency $\mathrm{AE}$, last two columns in Table 4. The parameter with the highest critical impact (and the largest experimental workload) on the absorbed photons is the absolute irradiation $I_{\mathrm{abs}}(\lambda)$, eqn (20). But this procedure has only to be performed once if the functionality of the light source is carefully monitored. For the presented results the linear variable filter leads to an illuminated energy of $6.2 \pm$ $1.1 \mathrm{~W} \mathrm{~m}^{-2}$ and the linear variable filter plus the UG11 filter to an irradiation of $3.6 \pm 1.3 \mathrm{~W} \mathrm{~m}^{-2}$. The average width of the illumination is $11 \mathrm{~nm} \pm 2 \mathrm{~nm}$, Fig. 1 and Table S6. $\dagger$

\subsection{Intensity and energy relations of the initiators}

This analysis of the data will focus on the Raman measurements. Details concerning the UV-vis monitoring are part of the subsection "Analysis of the UV-vis signal to curing correlation" (3.3). If a maximal optical absorption influence of the resin is assumed the variances of the data are smaller, see Tables $\mathrm{S} 4$ and S5. $\dagger$ But the tables within the ESI $\dagger$ contain all calculated results. The data obtained with maximal influence of the resin on the absorption are summarized in Table 5. Due to the limited number of measurements the detailed analysis mainly focuses on the results of TPO-L.

Effect of inhibition on $\boldsymbol{n}_{\mathbf{i}}$. For the bulk polymerization of commercial monomers the chain reaction is usually inhibited by storage stabilizers (hydroquinone monomethyl ether, MEHQ) and/or oxygen. ${ }^{41,44}$ Therefore the created radicals react with inhibitor/oxygen until overcompensation§ or consumption of the inhibiting species. Reworded, the radical concentration is nearly zero and the termination rate $R_{\mathrm{t}}$ is very high. All the steps leading to inhibition species and not resulting in chain reactions (within the solidification time) are defined as terminated, which are for example midchain radicals. ${ }^{28}$

For a discontinuous irradiation the number of radicals $R$ can increase if one flash leads to sufficient radicals (high rate $R_{\mathrm{INI}}$ by a huge number of photons) or if the reaction is slow/stable enough $(>15 \mathrm{~ms})$ to accumulate the radicals describable by an average radical balance $B_{\mathrm{A}}$,

$$
B_{\mathrm{A}}=\frac{\mathrm{d} R}{\mathrm{~d} t}=R_{\mathrm{INI}}-R_{\mathrm{t}}
$$

The differences in initial times (= photon number) $n_{\mathrm{i}}$, Table 5, Fig. S10 and S11, $\dagger$ cannot doubtlessly be assigned to a physical/chemical property of the system. The storage period influenced by manual mixing of the system defiantly influences the inhibition period. This effect has a higher impact for Raman analysis (deep curing) of the measurements performed using a LVF-HL plus UG11 filter, like TPO-L median $=344 \mathrm{~nm}$ respective $=364 \mathrm{~nm}$, Fig. 5 . The absorbance by the resin does not result in the consumption of the inhibitor because $n_{\mathrm{i}}$ increases for wavelengths smaller than the onset of the monomer absorbance. The analysis performed with maximal resin correction leads, for the TPO-L with LVF, to a nearly complete

$\S$ The formulation "overcompensation" describes the condition when, during a measured reduction in the monomer content, a significant amount of inhibition species is still present. 
compensation of the monomer absorption, Fig. 5. Additional experimental studies focusing on the inhibition period have to be performed for doubt-free conclusions and to calculate the reactivates of the initiators towards inhibitor compounds.

Effect of wavelength/intensity on photo-polymerization efficiency. The photo-polymerization efficiency $\Delta n$ is defined as the absorbed number of photons to cure the monomer, eqn (15). Effects that are considered/removed as much as possible are the resin absorbance, the differences in illuminated number of photons, the inhibition and the thickness variance. A significant difference can be observed between the flashlight and the UG11 measurements of TPO-L. This effect is, without any doubt, dependent on the number of absorbed photons per Raman measurement, Fig. S9. $\dagger$ Thereby the experiments with the flashlight (higher energy per flash) were recorded with 200 flashes per Raman step and the ones with the UG11 filter were performed with 2000 flashes. Hence an accumulation of the radicals, as assumed within the previous subsection, took place leading to a more than optimal amount of radicals (enhanced amount of side reactions, reduced reactivity towards acrylic double bonds, lower kinetic length of the chain reaction). This intensity related effect, Fig. 3 and 6, can also be demonstrated by comparing the LVF TPO-L measurements at 307 and $312 \mathrm{~nm}$ (the one with and the one without the UG11 filter) and the $\approx 370$ $\mathrm{nm}$ measurement with and without the UG11 filter, Table 4 and Fig. 1. The efficiency significantly increases with increasing wavelength median (linear variable filters) up to the absorbance onset of TPO-L at $\approx 410 \mathrm{~nm}$, Fig. 6 and S3. $\uparrow$ Thereby the resin absorbance and illumination differences are removed/strongly reduced so that an effective cleaving and/or a more selective reaction of the fragmentation intermediate are likely. A higher selectivity of "low energetic", low concentration benzoyl radicals was reported if the radicals are generated from surface modified $\mathrm{N}$-ZnO particles. ${ }^{16}$ A low amount of excited radicals seems to result in a smaller amount of side reactions, like the formation of midchain radicals, than the same radicals produced by the cleavage of $e . g$. $\alpha, \alpha$-dimethoxy- $\alpha$-phenylacetophenone. ${ }^{28}$ Within the cited ESR based study the curing rates of the model initiators (molecular and particular) were strongly different.

Nevertheless, the difference in the radical content (intensity) may not influence the monitored selectivity for midchain radical formation. Further studies would be interesting to optimize the selectivity and efficiency of chain reactions in bulk systems. Such controlling and monitoring of radical selectivity/ activity are normally not considered for industrial applications but as the results in this study confirm they may be important to optimize the whole procedure. The desired objective is an optimal curing result, with the smallest amount of power (number of photons) leading to well-defined solids (low amount of side reactions) and reproducible properties of the polymeric product. Both the initiators DMHA and TPO-L are highly reactive in the range up to the measured onsets in absorbance, Fig. S3. $\dagger$ The study further clearly confirms that overcompensation of the inhibitor (whereby the inhibitor is not fully consumed)§ as monitored for the UG11 measurements of TPOL, Table 5, leads to a significantly higher number of necessary photons to cure the resin. Such overcompensation within the centre of the curing area of course leads also to larger solids, Table 2, whereby it can be assumed that the kinetic length of the chain reaction (3.8) will be strongly dependent on the position relative to the maximal irradiation. Such effects may result in inhomogeneities in the final product.

To summarize, the presented technique allows the calculating, separating and analysing of the inhibition period and chain reaction. Together with the intensity and energy variation of the irradiation, multiple influencing factors of the photopolymerisation in bulk may be investigated. The number of measurements analysed in detail is too small for further wellfounded hypotheses but all results are accessible within Tables S2, S4 and S5.†

\subsection{Kinetic lengths of the chain reaction, quantum yields and efficiencies}

Average kinetic length of the chain reaction and quantum yields. If the inhibitors are not overcompensated $\S$ when monitored for high photon flux (see the previous subsection), the presented experimental technique allows separation of the initial period from the chain reaction. Hence, the minimal and maximal kinetic length of the chain reaction, CL, can be calculated by eqn (22) and Tables S4, S5 $\dagger$ and 4,

$$
\frac{N_{\mathrm{DB}}}{n_{\mathrm{f}}} \leq \mathrm{CL} \leq \frac{N_{\mathrm{DB}}}{\Delta n} .
$$

The maximal length, $\mathrm{CL}(\max )$, corresponds to the average number of propagation steps for one initialization event $=$ quantum yield. Generally the length has to be as large as possible. The differences in the consumed number of double bonds $N_{\mathrm{DB}}$ are similar for all cured samples so that conclusions concerning the initiation potential remain the ones of the previous subsection. For the chain lengths of free radical polymerization of multifunctional acrylate no literature values exist (the solid is cross-linked) but free radical polymerization of cyclohexyl acrylate (monofunctional) led to a chain length of 1120 (ref. 29), which is larger than but in the same range as the computed kinetic lengths of the chain reaction, Table 4.

Efficiencies. Especially for practical application, the kinetic length of the chain reaction is not sufficient to characterize the efficiency of an initiator-resin-light source combination. The relative absorption efficiency, $\mathrm{AE}$,

$$
\frac{n_{\mathrm{INI}}-n_{\mathrm{res}}}{n_{\mathrm{ill}}} \leq \mathrm{AE}\left(\leq \frac{n_{\mathrm{INI}}}{n_{\mathrm{ill}}}\right),
$$

takes the absorbance of the system into consideration and therefore combines the information within Fig. 5. The minimal relative absorption efficiency, $\mathrm{AE}(\mathrm{min})$, corresponds to the maximal influence of the resin as discussed within the manuscript, Table S5. $\dagger$ Especially for light sources with a narrow wavelength range this relative absorption efficiency will eminently influence the curing result of the initiator. For the used geometry, Scheme 1, bottom, $\mathrm{AE}(\max )$ 's larger than 0.85 result in a reductioon of the cascading effect $=$ more real 
incident angles, Table 3. Hence, the value describes a process dependent balance between $\operatorname{deep}(/$ through) and area curing.

Different efficiency like the irradiation corrected efficiency, Eff, can be defined by eqn (25),

$$
\mathrm{Eff}=\mathrm{CL} \times \mathrm{AE}=\mathrm{Eff}_{\text {nill }} / n_{\mathrm{ill}}=\mathrm{Eff}_{n_{\mathrm{INI}}} / n_{\mathrm{INI}},
$$

which is especially interesting if we are able to choose the illumination wavelength with a similar photon flux. Otherwise the relative efficiencies to the irradiated, $n_{\mathrm{ill}}$, and the absorbed, $n_{\text {INI }}$, number of photons would lead to a weighting and would be interesting to optimize the desired processes. Considering the resin absorbance, six efficiencies for both CL(max)'s by eqn (24) and (25) exist, Scheme S1. $\uparrow$ Thereby the irradiation corrected efficiency Eff is independent from the resin absorbance/ correction, Fig. S12. $\dagger$ This is a clear contrast to most values, for which a maximal influence of the monomer/polymer absorbance ( $\neq$ maximal values) and a minimal influence may be calculated by Tables $\mathrm{S} 2, \mathrm{~S} 4$ and $\mathrm{S} 5 . \dagger$

Within Fig. S12† the irradiation corrected efficiencies, Eff, for every initiator are visualized whereas within Fig. 7 the efficiency relative to Eff $_{n_{\mathrm{INI}}}$ is presented, which results in conditions nearer to application. Even for the irradiation corrected efficiency, Eff, the most effective reaction took place for the lowest number of photons at around $365 \mathrm{~nm}$. The differences between Eff $_{n_{\mathrm{INI}}}$, Fig. 7, of the measurements with and without the UG11 filter clearly demonstrate the advantage of this visualization that increased light intensities lead to a larger efficiency value. The initiator TPO-L is very efficient in the range of $350 \mathrm{~nm}$, even though the relative absorption efficiency is more than $20 \% / 50 \%$ smaller, Fig. 7 and S12, $\uparrow$ than below $320 \mathrm{~nm}$. In the authors opinion further and/or faster experiments have to be performed to verify or falsify the presented hypotheses or to conclude further hypotheses, especially concerning the reactivity of DMHA. After the presented verification of the UV-vis signal variation, the aligned setup, Scheme 1 , in combination with a LVF-UV-HL (230-500 nm) filter can be used to obtain faster results.

\section{Conclusions}

This study can be divided in the presentation of the experimental and analytical technique, the model experiments and the outlook/impact on the resin-initiator-light source relations.

The Raman UV-vis curing equipment is a combined monitoring system of photo-induced polymerization. It was proven within this study that the variations in the UV-vis signal are correlated with the reaction, even for influenced wavelength ranges (by scattering, interfering reflection). Hence, the UV-vis $45^{\circ}\left(\alpha_{\text {det }}=90^{\circ}\right)$ reflection setup, Scheme 1, bottom, can be used as a standalone system, without a Raman detection unit, to measure the solidification of even highly pigmented systems, Table 2 and Fig. S7.† On the basis of wavelength and intensity dependent measurements it was proven that both systems allow the performance of kinetic quantification with comparable intensities for photo-induced bulk polymerization. Due to its velocity and simplicity the UV-vis setup also has potential for online applications/monitoring, for example in printing machines. Depending on the geometry the resulting UV-vis signal will be less distorted and, therefore, easier to analyse than in the presented case.

Due to the availability of NIR-(/IR-) sensitive CCD detection units, or by using a low power wolfram illumination, it will also most likely be possible to monitor thermal induced polymerization by the presented analytical technique. The UV-vis monitoring system can also be combined with any well-known monitoring system, like IR (disperse or FT), disperse Raman system or even UV curing system, for rheometers. Our own research will use the $180^{\circ} \mathrm{UV}$-vis system, Scheme 1 , top, as a standalone technique with unique properties (especially velocity and statistical accuracy). The measuring time of a UVvis setup is shorter than the one of the Raman UV-vis setup. Hence, effects of storage time can be investigated/reduced. The resolution of the Raman data as presented in this study was usually 2000 flashes, whereas the one of the UV-vis data was 40 flashes. The used UV-vis receiver can store data in the range of $30 \mathrm{~ms}$ and, therefore, even much faster processes can be distinct. Additionally to the better resolution in time, the aligned UV-vis setup can vary the irradiation intensity by variation in the detection position (variation of the aperture position relative to the emitter, Scheme 1). The data analysis firstly presented within this study leads to absolute consumed monomers and absolute numbers of absorbed photons at a well-defined position of the curing setup. Hence, the average kinetic lengths of the chain reaction and different efficiencies were computed. The investigated model resin was a printable monomer mixture containing three different well-known initiators, Table 1. Even if only considering the absorbed number of photons (i.e. after removing the absorbance effects) the initiation potential of TPO-L is higher than the one of DMHA. Due to the analytical approach, the quantum yield of radical generation, and radical reactivity towards inhibitor consumption and towards acrylate double bonds may be investigated in the future. As expected, benzophenone as a Norrish type II initiator results in a low yield. ${ }^{44}$ Different additional results were obtained by the detailed analyses: TPO-L is not only the fastest tested initiator but also the impact of the monomer absorption is smaller than for DMHA. For this "versatile highly efficient liquid photo-initiator" ${ }^{22}$ the effect of resin absorption on deep curing is more pronounced. If using a LVF-UV-HL (230-500 nm) this effect of interfering absorbance can be further analysed.

Interestingly, the presented studies indicate that light with lower energy (up to the onset of the absorbance) or lower intensity results in larger average kinetic lengths of the chain reaction $>400$, that means higher reactivity towards the acrylic double bonds. This effect, based on the accumulation of radicals, could be further underlined by variation of the flash frequency for the TPO-L containing sample. The experiments without a filter (200 flashes per Raman) result in much larger average kinetic lengths of the chain reaction than the measurements with a UG11 filter (2000 flashes per Raman). Hence, a dependence on the flash frequency can be suggested, which results in a specific amount of produced radicals. The lifetime of these propagating radicals is larger than $15 \mathrm{~ms}$, so 
that a more than optimal amount of radicals is possible. Further experiments are necessary to underline this hypothesis. All results are accessible within Tables $\mathrm{S} 2, \mathrm{~S} 4$ and $\mathrm{S} 5 \dagger$ to allow performing future interpretations of the data.

The analytical methods, as firstly presented in detail within this contribution, could be essential for the development/ optimization of inhibitor-resin-light source combinations. Both parts of the setup (Raman and UV-vis) are exchangeable/ variable. For example, microscopic optics results in the possibility to investigate several locally distributed factors, like the mobility of propagation/inhibiting species and micro gel formation. Wavelength dependent analysis can be used to investigate the quantum yield for triplet state formation or radical reactivity (difference in absorbance can be in situ monitored). The variability of the fibre optical system e.g. to choose the wavelengths (visible and IR) by light source and filter variations can also be combined with temperature dependent analysis (e.g. temperature variation of the sample holder and IRfree illumination with well-defined energy and known intensity).

From the aspect of application, the presented study may be useful to develop LED simulations with comparable intensity, which may be essential to develop selective initiators for this novel kind of light source. For example, recently $365 \mathrm{~nm}$ high power UV-LED curing units ${ }^{\mathbf{3 8}}$ achieved market readiness. Power outputs of $70 \mathrm{~kW} \mathrm{~m}{ }^{-2}$ (365 $\mathrm{nm}$ ) can be emitted by using water cooled arrays of diodes. ${ }^{38}$ The tested xenon flash light has an output of only around $25 \mathrm{~W}$ resulting in an energy on the sample of around $115 \mathrm{~W} \mathrm{~m}^{-2}$ (255 nm to $950 \mathrm{~nm}$ ) or $5 \mathrm{~W} \mathrm{~m}^{-2}$ $(367.5 \mathrm{~nm} \pm 6 \mathrm{~nm})$. By considering a maximal area of $24 \mathrm{~mm}^{2}$, less than $0.001 \%$ o $(1 \mathrm{ppm})$ of the light reaches the sample. Interestingly, this is exactly the amount that was calculated in Schmitt, 2011. ${ }^{14}$ One flash corresponds to $8 \mu$ s of illumination time so that the sample is exposed to $0.075 \mathrm{~J} \mathrm{~m}^{-2}$ by the xenon light and illuminated with $0.56 \mathrm{~J} \mathrm{~m}^{-2}$ by the high power LED array. Note that the used xenon light source is around $10^{2}$ times less intensive than a commercial lab curing unit, see subsection 3.6 (without considering different energy distributions). But it is relatively simple to implement different light sources (continuous emission, higher output or enhanced collecting). Even the effect of UV-B irradiation can be analysed by such equipment if an adjustable bandpass filter with a different working range, like LVF-UV-HL (230-500 nm), is used. Then, the effect of the resin can be investigated more in detail. At the moment such studies below $360 \mathrm{~nm}$ are impossible to perform by using LED radiation.

\section{Acknowledgements}

I want to thank F. Heib and R. Hempelmann for their support.

\section{Notes and references}

1 I. I. Abu-Abdoun and A. Aale, Eur. Polym. J., 1993, 29, 14451450.

2 J. Barth, M. Buback, P. Hesse and T. Sergeeva, Macromolecules, 2010, 43, 4023-4031.
3 M. Buback, L. H. Garciarubio, R. G. Gilbert, D. H. Napper, J. Guillot, A. E. Hamielec, D. Hill, K. F. Odriscoll, O. F. Olaj, J. C. Shen, D. Solomon, G. Moad, M. Stickler, M. Tirrell and M. A. Winnik, J. Polym. Sci., Part C: Polym. Lett., 1988, 26, 293-297.

4 M. Buback and R. A. Laemmel, Macromol. Theory Simul., 1997, 6, 145-150.

5 O. Elizalde, J. R. Leiza and J. M. Asua, Macromol. Symp., 2004, 206, 135-148.

$6 \mathrm{~J} . \quad$ P. Fouassier, D. Ruhlmann and A. Erddalane, Macromolecules, 1993, 26, 721-728.

7 A. J. Hoffman, G. Mills, H. Yee and M. R. Hoffmann, J. Phys. Chem., 1992, 96, 5546-5552.

8 B. Kraeutler, H. Reiche, A. J. Bard and R. G. Hocker, J. Polym. Sci., Part C: Polym. Lett., 1979, 17, 535-538.

9 M. Schmitt, J. Wagner, G. Jung and R. Hempelmann, J. Colloid Interface Sci., 2007, 311, 425-429.

10 K. Prajapati and A. Varshney, J. Polym. Res., 2006, 13, 97-105. 11 A. Endruweit, M. S. Johnson and A. C. Long, Polym. Compos., 2006, 27, 119-128.

12 M. Skrifvars, P. Niemela, R. Koskinen and O. Hormi, J. Appl. Polym. Sci., 2004, 93, 1285-1292.

13 M. Schmitt, S. Kuhn, M. Wotocek and R. Hempelmann, $Z$. Phys. Chem., 2011, 225, 297-311.

14 M. Schmitt, Macromol. Chem. Phys., 2011, 212, 1276-1283.

15 M. Schmitt, R. Schulze-Pillot and R. Hempelmann, Phys.

Chem. Chem. Phys., 2011, 13, 690-695.

16 M. Schmitt, Macromol. Chem. Phys., 2012, 213, 1953-1962.

17 M. Schmitt, Nanoscale, 2015, 7, 9532-9544.

18 K. L. Mendoza, A. Ortega and N. S. Kim, J. Electron. Mater., 2015, 44, 784-791.

19 M. Han, W. Lee, S. K. Lee and S. S. Lee, Sens. Actuators, A, 2004, 111, 14-20.

20 I. Cooperstein, M. Layani and S. Magdassi, J. Mater. Chem. C, 2015, 3, 2040-2044.

21 C. Dietlin, S. Schweizer, P. Xiao, J. Zhang, F. Morlet-Savary, B. Graff, J.-P. Fouassier and J. Lalevee, Polym. Chem., 2015, 6, 3895-3912.

22 C. Decker and K. Moussa, J. Appl. Polym. Sci., 1987, 34, 16031618.

23 P. M. Johnson, J. W. Stansbury and C. N. Bowman, Macromol. React. Eng., 2009, 3, 522-528.

24 C. Decker and K. Moussa, Macromol. Chem. Phys., 1988, 189, 2381-2394.

25 C. Decker, K. Moussa and T. Bendaikha, J. Polym. Sci., Part A: Polym. Chem., 1991, 29, 739-747.

26 M. D. Dickey and C. G. Willson, AIChE J., 2006, 52, 777-784.

27 A. K. O'Brien and C. N. Bowman, Macromolecules, 2006, 39, 2501-2506.

28 M. Schmitt, Analyst, 2013, 138, 3758-3770.

29 B. Yamada, M. Azukizawa, H. Yamazoe, D. J. T. Hill and P. J. Pomery, Polymer, 2000, 41, 5611-5618.

30 G. Allegrone, I. Tamaro, S. Spinardi and G. Grosa, J. Chromatogr. A, 2008, 1214, 128-133.

31 S. Aprile, E. Del Grosso and G. Grosa, Xenobiotica, 2011, 41, 212-225. 
32 H. Gallart-Ayala, O. Nunez, E. Moyano and M. T. Galceran, J. Chromatogr. A, 2011, 1218, 459-466.

33 M. Hefnawy, Advances in Food Protection Series A: Chemistry and Biology, Springer VCH, 2010.

34 T. Jung, T. J. Simat and W. Altkofer, Food Addit. Contam., Part A, 2010, 27, 1040-1049.

35 T. Jung, T. J. Simat, W. Altkofer and D. Fugel, Food Addit. Contam., Part A, 2013, 30, 1993-2016.

36 A. K. Malik, C. Blasco and Y. Pico, J. Chromatogr. A, 2010, 1217, 4018-4040.

37 T. Rothenbacher, M. Baumann and D. Fugel, Food Addit. Contam., 2007, 24, 438-444.

38 Heraeus Noblelight GmbH, The Heraeus Noblelight UV-LED portfolio, 2014.
39 M. Schmitt and F. Heib, RSC Adv., 2014, 4, 17639-17647.

40 R. S. P. Teixeira, R. J. Correa, A. Belvino and R. S. V. Nascimento, J. Appl. Polym. Sci., 2013, 130, 24582467.

41 G. Moad and D. H. Solomon, The Chemistry of Radical Polymerization, Elsevier B.V., 2006.

42 BASF, Ciba ${ }^{\circledR}$ DAROCUR ${ }^{\circ}$ 1173, 2014.

43 O. Paul, A. Quosig, T. Bauer, M. Nittmann, J. Bartschke, G. Anstett and J. A. L'Huillier, Appl. Phys. B: Lasers Opt., 2007, 86, 111-115.

44 J.-P. Fouassier, Photoinitiation, Photopolymerization, and Photocuring: Fundamentals and Applications, Hanser Gardner Pubns, 1995. 